\title{
Simulation-Based Training Improves Student Assessment of Oral Feeding Skills in Preterm Infants
}

\author{
Courtney K. Broadfoot \\ University of Wisconsin-Madison, ckbroadfoot@gmail.com \\ Julie M. Estis \\ University of South Alabama, jestis@southalabama.edu \\ DOI: https://doi.org/10.30707/TLCSD4.3/FVMX2142
}

Follow this and additional works at: https://ir.library.illinoisstate.edu/tlcsd

Part of the Communication Sciences and Disorders Commons, and the Educational Technology Commons

\section{Recommended Citation}

Broadfoot, Courtney K. and Estis, Julie M. (2020) "Simulation-Based Training Improves Student Assessment of Oral Feeding Skills in Preterm Infants," Teaching and Learning in Communication Sciences \& Disorders: Vol. 4: Iss. 3, Article 8. DOI: https://doi.org/10.30707/TLCSD4.3/FVMX2142

Available at: https://ir.library.illinoisstate.edu/tlcsd/vol4/iss3/8

This Scholarship of Teaching and Learning Research is brought to you for free and open access by ISU ReD: Research and eData. It has been accepted for inclusion in Teaching and Learning in Communication Sciences \& Disorders by an authorized editor of ISU ReD: Research and eData. For more information, please contact ISUReD@ilstu.edu. 


\title{
Simulation-Based Training Improves Student Assessment of Oral Feeding Skills in Preterm Infants
}

\begin{abstract}
Advancements in medical technology have contributed to increased rates of preterm birth. Prematurity places infants at high risk for feeding difficulties, however. Early identification and assessment of preterm infant dysphagia is critical to maximize nutrition and hydration, feeding safety, and growth and development. The purpose of this study was to assess the ability of a simulation-based training toolto increase non-health care and entry-level clinical student sensitivity to signs of feeding distress in preterm infants. Data were collected from 60 students (20 masters-level Speech-Language Pathology, 20 undergraduate nursing, 20 undergraduate non-health care) in a pre-test/post-test design. All participants completed a brief simulation training protocol, and accuracy percentages were calculated based on their ability to determine the following: physiological and behavioral signs of feeding distress, oral feeding skill level (OFS), and clinical recommendation for further feeding evaluation. Our results revealed that this simulation-based training improved the identification of behavioral $(p<0.001)$ and physiological $(p<$ $0.001)$ signs of feeding distress, OFS level $(p<0.001)$, and ability to make appropriate clinical recommendations $(p<0.001)$. This study has identified a successful method to effectively train entry-level clinical and non-clinical students to screen feeding skills in preterm infants. This training approach has the potential to improve identification of feeding distress and to recognize the need for a dysphagia evaluation to optimize clinical outcomes in this fragile population.
\end{abstract}

\section{Keywords}

Dysphagia, preterm infant, simulation, assessment 


\section{Introduction}

While advancements in medical technology have substantially improved clinical outcomes, preterm birth rates have risen world-wide over the last decade (Blencowe et al., 2012; Liu et al., 2016). For instance, one out of every ten births in the U.S. are preterm (Hamilton et al., 2015). Infants born prematurely face increased risks of developmental concerns and subsequent need for healthcare (Bird et al., 2010; Glass et al., 2015; Petrou, 2005; Schmitt et al., 2016). More than 26\% of preterm infants demonstrate difficulty feeding and swallowing, also known as dysphagia (Mercado-Deane et al., 2001). Dysphagia increases the risk for malnutrition, dehydration, and aspiration, which can lead to aspiration pneumonia and other complex respiratory conditions (Eun Uhm et al., 2013; Groher \& Crary, 2010; Kakodkar \& Schroeder, 2013; Lefton-Greif, 2008; Volpe, 2008). Further, feeding ability is a major concern for parents of infants in the Neonatal Intensive Care Unit (NICU) and significantly contributes to maternal psychological distress (Park et al., 2016).

To optimize feeding outcomes and decrease the risk of health concerns in this fragile population, caution is taken when transitioning preterm infants to oral feeding. This transition requires careful medical monitoring, and a specialized and trained clinical care team to recognize distress signals of preterm infants and to accurately assess their oral feeding readiness. Speech-language pathologists are brought into the healthcare team to assist the nursing staff and caregivers in this oral feeding transition. Speech-language pathologists provide education to the nursing staff and caregivers and are able to assess for signs of feeding safety to maximize oral feeding success. For instance, during oral intake, infants display behavioral and physiological signs to indicate distress (Ferguson et al., 2015; Groher \& Crary, 2010). Physiological responses are automatic reactions to indicate feeding distress and include coughing, apnea, desaturation, color changes, hypoventilation, and bradycardia (Koenig et al., 1990; Mathew, 2011; Thach, 2007; Thoyre \& Carlson, 2003a., 2003b.). Additionally, behavioral responses that are adaptive or compensatory behaviors indicating feeding distress include drooling, loud breathing and swallowing, gulping, or abrupt muscle tone changes (Giambra \& Meinzen-Derr, 2010; Liaw et al., 2005; Thoyre et al., 2005).

In addition to monitoring behavioral and physiological signs of feeding safety, a volume-based, objective Oral Feeding Skill (OFS) assessment tool (Figure 1) has been developed and validated (Lau \& Smith, 2011, 2012). Briefly, the OFS acts as a triage tool to categorize the feeding skill level of the preterm infant based on volume measurements taken during the oral feeding session. This tool defines the oral feeding skill level as the combined proficiency (PRO, \%), which is a measurement taken at the beginning of the feed and the rate of milk transfer (RT; $\mathrm{mL} / \mathrm{min}$ ) which is measured over the entire feed. The PRO measurement captures the infants actual feeding ability, whereas the RT indicates the endurance of the infant during oral feeding. Infant scores are categorized into four OFS Levels based on their PRO and RT values, which indicate the feeding skill advancement of the infant. Thus, this tool can provide valuable insight to the oral feeding readiness of the infant. 
Figure 1. Oral Feeding Skills Assessment Tool as obtained from Lau \& Smith, 2012.

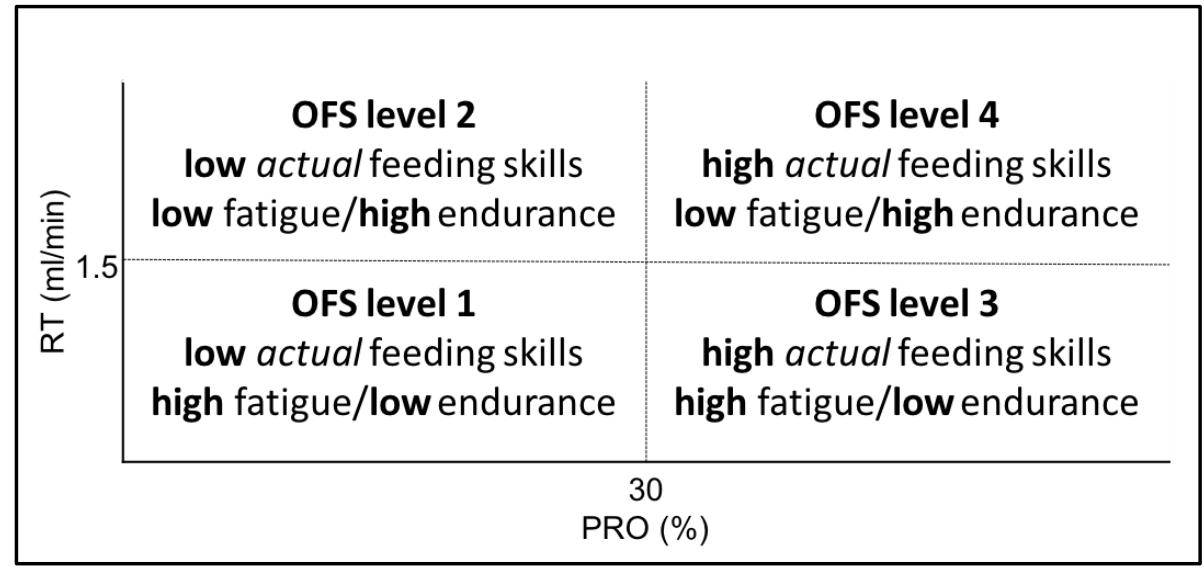

Determining preterm infant feeding safety is complex, and the need for specialized health-care training in this area is high. Because the speech-language pathology and nursing team and the caregivers are the most involved in the feeding process, it is critical to ensure these individuals are well-equipped to accurately assess infant feeding safety. This is a current challenge, however, because there is no standardized training method to educate novice clinicians or caregivers to assess preterm infant feeding ability. A standardized educational tool could be impactful in providing effective training to novice clinicians, as well as educate caregivers to feel more in control of their infant's care. Simulation-based education, specifically with the use of high-fidelity manikins, offer a unique opportunity to teach various techniques and training tools, bolster foundational knowledge of complex populations, and improve comfort levels of students (Dudding et al., 2019). Simulation-based education is an accepted educational practice across many disciplines and recent literature shows promise for its utility to improve management and assessment of dysphagia (Benadom \& Potter, 2011; Jansen et al., 2015; Miles et al., 2016; Ward et al., 2015). Specifically, Ferguson and Estis (2018) determined that video-recorded patient simulation training was as effective as traditional didactic instruction to increase student ability to assess preterm infant feeding skills (Ferguson \& Estis, 2018). These students were familiar with assessing patient vital signs and making clinical recommendations. Thus, to expand on this previous literature and to more closely represent novice clinicians and caregivers, this study included participants without previous knowledge of preterm infant dysphagia care, assessment, or management.

Thus, the purpose of this study was to determine if a simulation-based training protocol would be a successful method to educate novice students to accurately assess feeding safety in preterm infants. The study asked the following research questions: 1) Is this simulation-based education an effective method to improve student abilities to identify behavioral and physiological markers of feeding distress using the OFS assessment tool, and make clinical recommendations? 2) Are there differences in percent correct scores prior to or following training among the three student groups? We hypothesized that this simulation-based training would improve the accuracy of identification of feeding distress signs (behavioral and physiological), increase the ability to use the OFS assessment tool, and improve the accuracy of clinical recommendations. 


\section{Methodology}

Study Design. A pre-/post-training within- and between-subjects design was used to determine the effect of this simulation-based training on participants' ability to 1) identify physiological and behavioral signs of feeding distress, 2) use an objective oral feeding skill assessment tool, and 3) make appropriate clinical recommendations. Specifically, participants were asked to make a clinical decision to either continue feeding the infant or to stop the oral feeding and refer for a comprehensive swallowing evaluation. Prior to the study, participants completed written consent forms and all procedures were approved by the University of South Alabama Institutional Review Board.

Participants. Graduate level speech-language pathology students $(n=20)$ and undergraduate level nursing students $(n=20)$ and non-health care students $(n=20)$ participated in this study $(N=60)$. Non-health care students were defined as undergraduate students with non-health care related undergraduate majors, such as accounting, business, and computer sciences. All participants were English speaking, at least 19 years old, and enrolled at the University of South Alabama. Participants were excluded if they were or have been a parent of an infant in the NICU, or if they reported more than two days of clinical experience in the NICU. Demographic data for the participants is found in Table 1.

\begin{tabular}{|lll|}
\hline Table 1 & \\
$\begin{array}{l}\text { Self-reported demographic information of the participants } \\
\text { (standard deviation) and sex. }\end{array}$ & \\
\hline Student Group & Mean Age (Std. Dev) & Sex (F/M) \\
\hline Non-health care & $22.5(.82)$ & $16 / 4$ \\
Nursing & $22.1(.77)$ & $17 / 3$ \\
Speech-language pathology & $23.7(.21)$ & $20 / 0$ \\
\hline
\end{tabular}

Procedures. All study procedures were completed in the Health Sciences Building at the University of South Alabama. The orientation and training videos were projected on a large screen and viewed in a classroom setting as a group and participants completed pre- and post-testing score sheets individually (see Table 2). Participants watched a three-minute orientation video that described the experimental task and explained the components of the cardiopulmonary monitor. To determine participant assessment abilities at baseline, a pre-test was administered. The pre-test consisted of two, two-minute, simulation-based scenario videos. Participants were instructed to identify physiological and behavioral signs of feeding distress, use the OFS assessment tool, and make a clinical recommendation to either continue feeding the infant or to stop the feeding session and refer for a comprehensive swallow evaluation. Participants then participated in a brief, 60minute simulation-based training protocol. All pre-test and post-tests were administered individually and there was no time limit to complete the testing. However, no participant took longer than five minutes to complete each simulation-based scenario. For the post-test, participants viewed six, two-minute simulation-based feeding scenario videos and were asked to identify and document physiological and behavioral signs of feeding distress, use the OFS assessment tool, and provide a clinical recommendation for each video feeding scenario. 


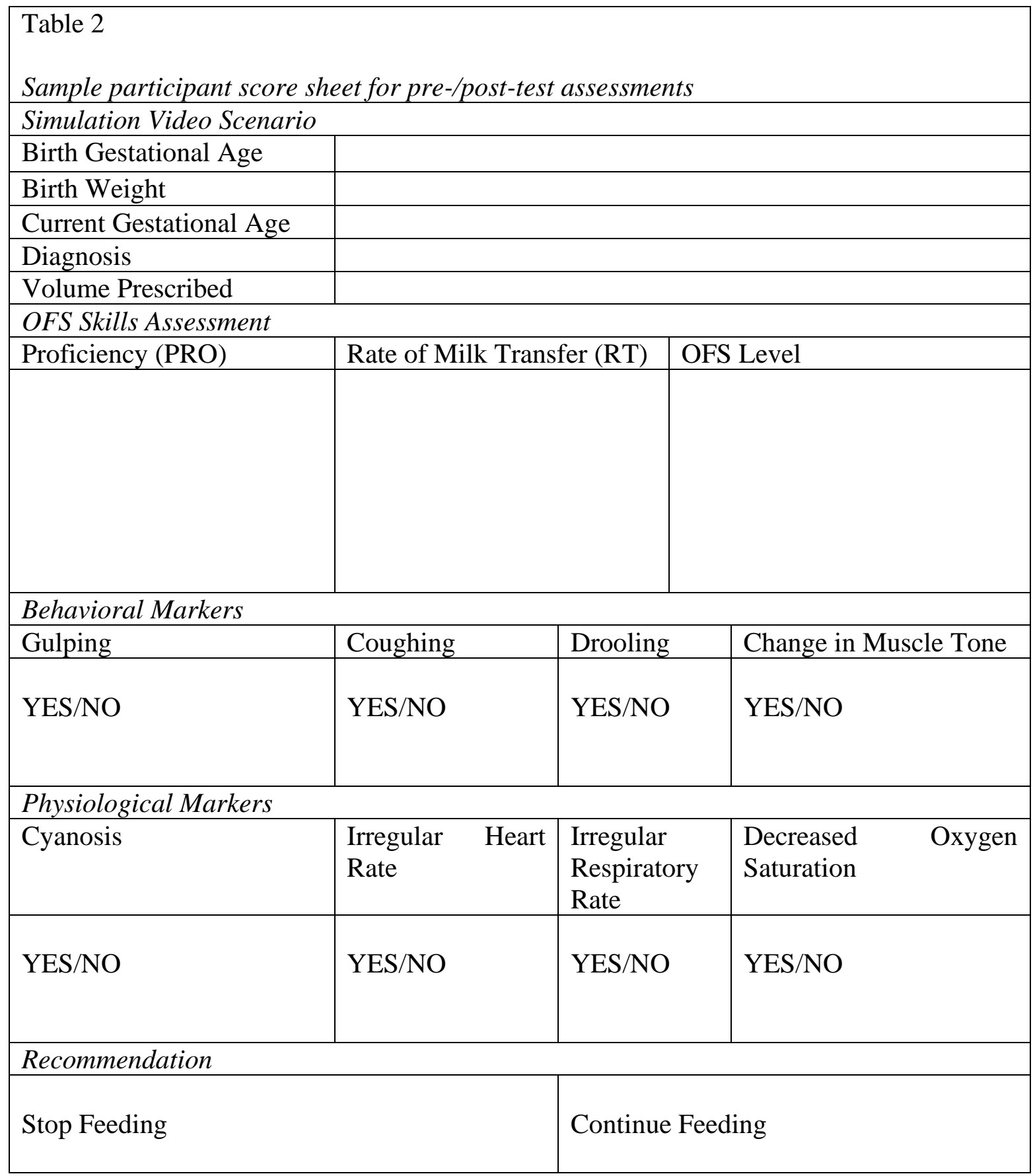

Simulation-based Training. This simulation-based training was administered with a thirtyminute, pre-recorded, voice-over video presentation that was modified from previous work in our laboratory (Ferguson \& Estis, 2018). The simulation-based training described the overall immaturity of preterm infant biological systems that may be contributing to feeding difficulties, provided descriptions and examples of physiological and behavioral distress signs of feeding difficulty in preterm infants, demonstrated use of the OFS assessment tool including calculation examples, and provided a clinical guide to integrate findings from the assessment to determine need for a comprehensive feeding evaluation. All participants were provided a printed version of 
the simulation-based training presentation as well as time to ask questions.

Simulation-based Feeding Scenario Videos. To simulate preterm infant feeding scenarios, a high-fidelity human patient mannequin (HAL® model S3010) was programmed to exhibit physiological and behavioral signs of feeding distress and the simulation feeds were video recorded. The movements of HAL® were manipulated to include cyanosis (skin turning blue), chest rise, spontaneous breathing, rate and depth of respiration, and movement of limbs and muscle tone. A split-screen was used to display the cardiopulmonary monitor to include the respiratory rate (located on the bottom of the screen and represented in blue), heart rate (located on the top of the screen and represented in green), and oxygen saturation level (located in the middle of the screen and represented in red). These simulation-based feeding scenario videos were modified from those described by Ferguson \& Estis. (2018) to include an animated bottled and timestamp of the feed (Figure 2). Each video was edited to present the feed in 30 second intervals at the following times: one minute, five minutes, fifteen minutes, and twenty minutes. This allowed participants to observe and assess the infant at the beginning of their feed, during the middle of the feed, and at the end of the feed. The animated bottle allowed for real-time analysis of volume changes to more accurately represent a realistic feeding scenario. Participants viewed each simulation-based feeding scenario and were given five minutes to identify and document feeding distress signs, calculate the OFS level, and provide a clinical recommendation. A brief case-history including primary diagnosis, gestational age, birth weight, and the formula volume prescribed were provided with each simulation-based feeding scenario video.

Figure 2. Simulation-based Feeding Scenario Video Illustration

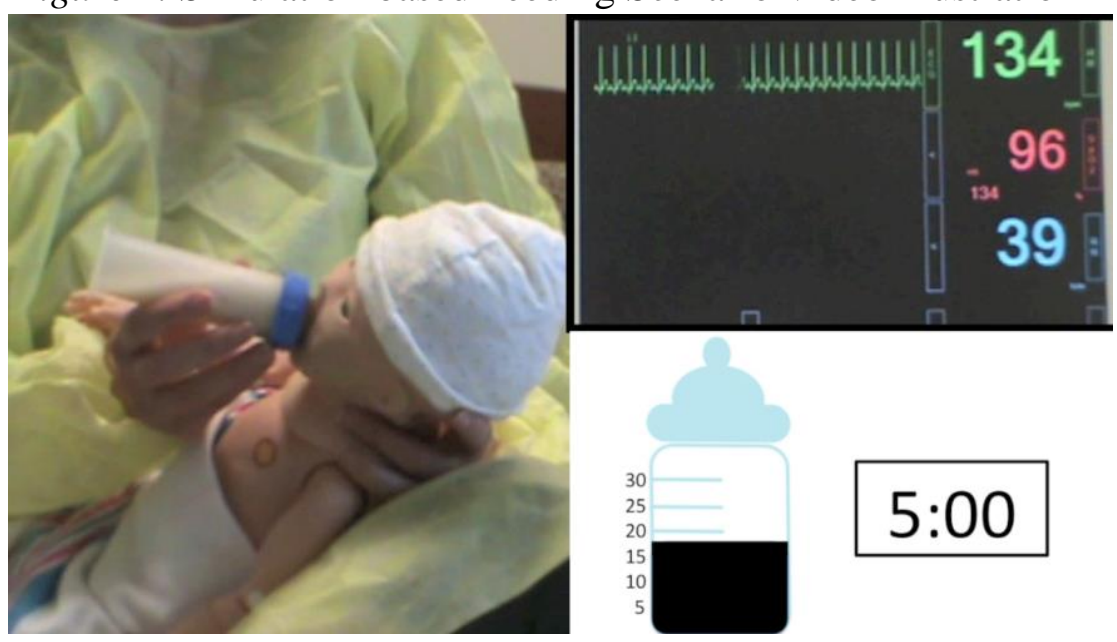

Outcome Variables. The primary outcome variables were: average percent accuracy values within each student group for identifying behavioral and physiological signs of feeding distress $(\max =100 \%)$, OFS level calculations $(\max =100 \%)$, and clinical recommendation to either continue feeding or to stop feeding and refer for a swallow evaluation $(\max =100 \%)$.

Statistical Analyses. All statistical analyses were conducted with SigmaPlot version 12.5 from Systat Software, Inc., San Jose California USA, www.systatsoftware.com. Table 3 illustrates means and standard error of the mean for all outcome variables. To determine if there were 
differences in accuracy scores before and after training and if there were differences between groups, a two $x$ three repeated measures analysis of variance (ANOVA) was carried out with factors of timepoint (pre/post-training) and student group (speech-language pathology, nursing, non-health care). All data were tested for normality using the Shapiro-Wilk test (Shapiro \& Wilk, 1965) and assessed for equal variances using the Brown-Forsyth analysis (Brown \& Forsythe, 1974). This ensured the assumptions of equal variance and normality of the ANOVA were upheld. When significant effects were found, post-hoc analysis were performed using Holm-Sidak methods (Holm, 1979). The critical level of significance was set a priori at $p<0.05$.

\section{Results}

Behavioral Signs of Feeding Distress. Illustrated in Figure 3A, there was a significant interaction between training and student group $[\mathrm{F}(2,57)=4.893, p=0.011]$. At the pre-training timepoint, nursing students had significantly higher accuracy percentages than non-health care students $(p=$ 0.002). There were no other significant differences at baseline. After training, however, all student groups significantly improved: non-healthcare ( $p<0.001)$, nursing $(p<0.001)$, speech-language pathology $(p<0.001)$. Additionally, there were no significant differences in accuracy percentages between student groups after training.

Physiological Signs of Feeding Distress. Statistical analysis revealed there were no interaction effects, so data were collapsed to appropriately represent the results (Figure 3B). Analysis found an effect of training $[\mathrm{F}(1,57)=101.774, p<0.001]$ and an effect of group $[\mathrm{F}(2,57)=3.223, p=$ 0.047]. Regardless of training, speech-language pathology students demonstrated higher average accuracy scores compared to non-health care students $(p=0.041)$. All student groups significantly improved after training $(p<0.001)$.

Oral Feeding Skill Assessment Tool. There was a significant interaction between training and student group $[\mathrm{F}(2,57)=4.912, \mathrm{p}=0.011]$ (Figure 3C). There were no significant differences in accuracy scores between student groups at the pre-training timepoint. All student groups significantly improved after training: non-healthcare $(p<0.001)$, nursing $(p<0.001)$, speechlanguage pathology $(p<0.001)$. After training, speech-language pathology students demonstrated higher accuracy scores than non-health care $(p<0.001)$ and nursing students $(p=0.001)$; however, all student groups had average accuracy scores above $89 \%$ post-training (Table 3 ).

Clinical Recommendation. There were no interaction effects found and the data were collapsed to illustrate these results. Analysis found a significant effect of training $[\mathrm{F}(1,57)=81.235, p<$ $0.001]$ and an effect of student group $[\mathrm{F}(2,57)=5.762, p=0.005]$ (Figure 3D). The speechlanguage pathology students demonstrated higher accuracy to make appropriate clinical recommendations compared to the non-health care $(p=0.009)$ and nursing students $(p=0.015)$. All student groups significantly improved after training $(p<0.001)$. 
Figure 3. Accuracy percentages for the identification and documentation of the following outcome variables: (A) behavioral signs of feeding distress, (B) physiological signs of feeding distress, (C) calculations of Oral Feeding Skill level (OFS), and (D) clinical recommendations to continue/stop infant feeding. $(*$ indicates $\mathrm{p}<0.05$, ** indicates $\mathrm{p}<0.001)$.

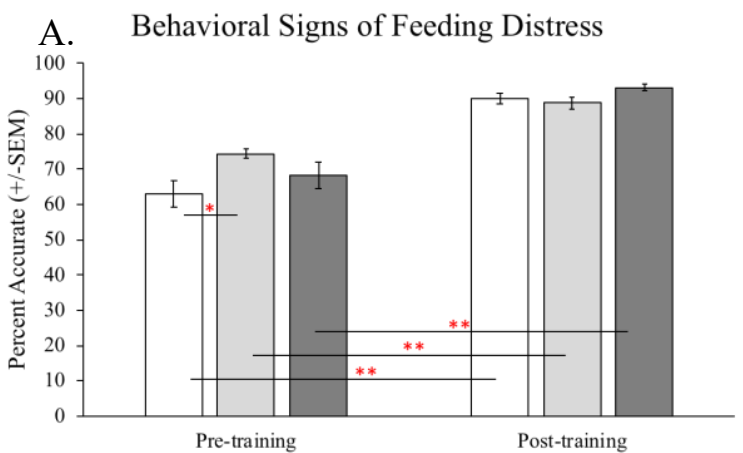

$\square N H C \square R N$ aSLP

C. Oral Feeding Skills Assessment Tool

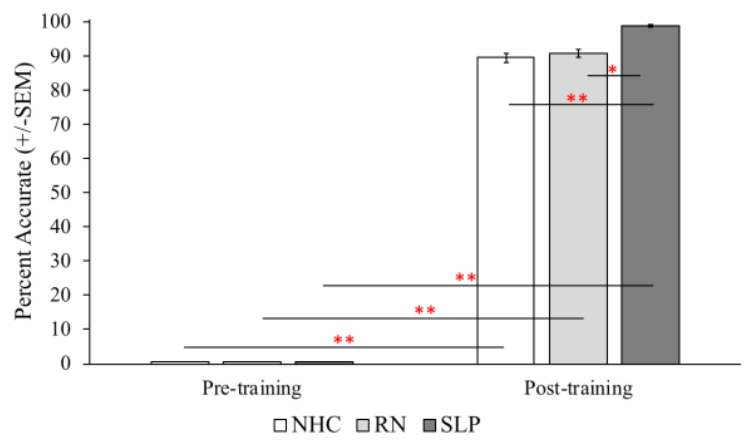

B. Physiological Signs of Feeding Distress
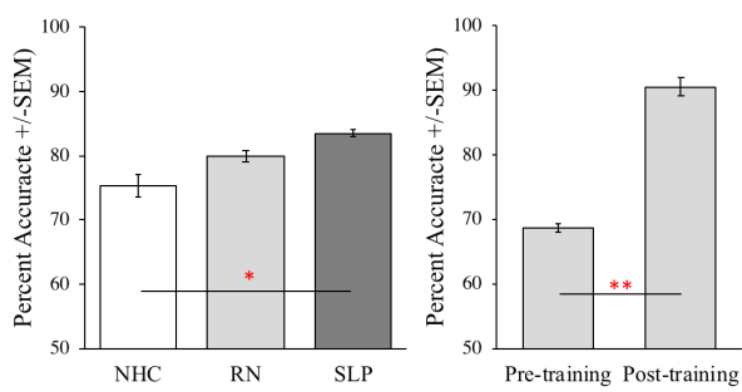

D.
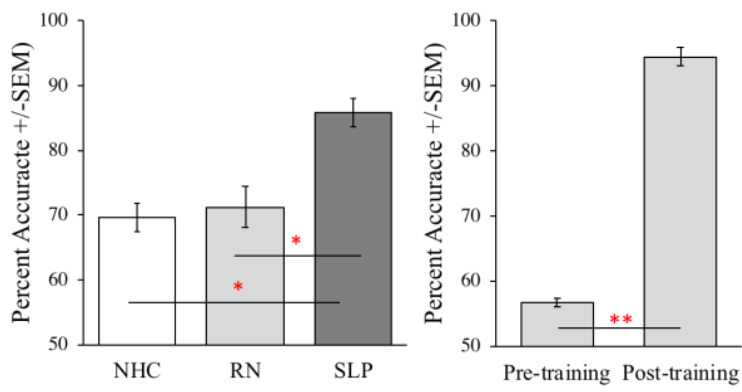

\begin{tabular}{|c|c|c|c|c|c|}
\hline \multicolumn{6}{|c|}{$\begin{array}{l}\text { Calculated means (standard error of the mean) for percent accuracy scores of non-health } \\
\text { care }(N H C) \text {, nursing }(R N) \text {, and speech language pathology }(S L P) \text { students. }\end{array}$} \\
\hline & $\begin{array}{l}\text { Student } \\
\text { Group }\end{array}$ & Behavioral & Physiological & OFS Level & Clinical Rec. \\
\hline \multirow[t]{3}{*}{ Pre-training } & $\mathrm{NHC}$ & $63.1(1$ & 63.8 & 0.0( & $47.5(2.40)$ \\
\hline & RN & $74.4(1.05)$ & $70.6(1.09)$ & $0.0(0)$ & $50.0(2.37)$ \\
\hline & SLP & $68.1(0.89)$ & $71.9(1.21)$ & $0.0(0)$ & $72.5(2.26)$ \\
\hline \multirow[t]{3}{*}{ Post-training } & NHC & $90.0(2.37)$ & $87.1(2.52)$ & $89.6(1.06)$ & $91.7(1.31)$ \\
\hline & $\mathrm{RN}$ & $88.8(2.45)$ & $89.1(2.53)$ & $90.7(0.92)$ & $92.5(0.94)$ \\
\hline & SLP & $93.1(0.69)$ & $95.2(0.57)$ & $98.9(0.43)$ & $99.2(0.46)$ \\
\hline
\end{tabular}




\section{Discussion}

We hypothesized that this simulation-based training protocol would be an effective method to train graduate level speech-language pathology and undergraduate level nursing and non-health care students to accurately identify signs of feeding distress, use an objective assessment tool to categorize infant feeding skill level, as well as make appropriate clinical recommendations regarding infant feeding safety. The results of this study indicated that all student groups (nonhealth care, nursing, and speech-language pathology) demonstrated significant improvement in accuracy scores for all outcome variables post-training. The speech-language pathology students out-performed their peers (nursing and non-health care) in their ability to use the OFS tool and make clinical recommendations. Additionally, the speech-language pathology students more accurately identified physiological signs of feeding distress compared to non-health care students after training. We expect that their graduate-level status as well as prior knowledge of dysphagia from undergraduate coursework may have contributed to this success as they are the least novice of the three study groups. Importantly, all three student groups achieved exceptionally high accuracy scores for all outcome variables after training. This study confirmed this simulationbased training as an effective and efficient method for educating both students in clinical and nonclinical fields to assess aspects of swallowing safety and efficiency that are critical to the success of preterm infants.

Current Findings in Relations to Other Work. The results of this study are in agreement with the findings of Ferguson and Estis (2018). The authors found that simulation-based training, when compared to lecture-based education, was an effective method to train nursing and speechlanguage pathology students to identify physiological and behavioral signs of feeding distress, calculate OFS level, and make appropriate clinical recommendations. The results of the present study has affirmed the use of simulation-based training to identify preterm infant feeding distress signals and to recognize the need for additional swallow assessment.

Study Limitations and Clinical Implications. An inherent limitation to simulation-based research is the ability to generalize these findings to human patient scenarios. The simulation feeding scenarios from this study did not include interactions with preterm infants in the NICU. Carefully considering the medical fragility of the preterm infant population, mitigating potential risks to this patient group is exceptionally important. Thus, simulation-based education creates an opportune exposure for clinicians and caregivers to gain education and experience with complex medical information without generating patient risk. Therefore, the simulation-based infant feeding scenarios created for this study were purposefully designed to mimic preterm infant feeding scenarios as realistically as possible.

Another acknowledged limitation to this study is that the demographic data (Table 1) and the college-level educational status of the participants in this study does not directly represent the general population and we did not include caregivers of infants in the NICU. Thus, to determine if this simulation-based training would increase skills of practicing clinicians or caregivers of infants in the NICU, future research is warranted. However, we believe this was a necessary first step, and the findings of this work have impactful clinical relevance. This work will guide future studies to ensure the simulation-based training practices are optimal across clinical and classroombased educational settings. 


\section{Conclusion}

This study identified the effectiveness of a simulation-based training to educate speech-language pathology, nursing, and non-health care students to identify preterm infant feeding and swallowing difficulties, use an objective assessment tool, and make appropriate clinical recommendations regarding infant feeding care. Future research to include practicing clinicians and caregivers of infants in the NICU is warranted as this training tool has the potential to be a useful educational tool for individuals responsible for the feeding and care of this fragile population. Utilizing simulation-based training in the clinical setting to improve novice clinician and caregiver abilities to identify potential feeding problems in the preterm infant population would lead to improved feeding outcomes and optimized clinical care for these patients.

\section{Disclosures}

Procedures were approved by the University of South Alabama Institutional Review Board.

Courtney Broadfoot is employed by the University of Wisconsin-Madison and has no other relevant financial relationships to disclose. She has no relevant non-financial relationships to disclose.

Julie Estis is employed by the University of South Alabama and has no other relevant financial relationships to disclose. She has no relevant non-financial relationships to disclose.

\section{References}

Benadom, E. M., \& Potter, N. L. (2011). The use of simulation in training graduate students to perform transnasal endoscopy. Dysphagia, 26(4), 352-360. https://doi.org/10.1007/s00455-010-9316-y

Bird, T. M., Bronstein, J. M., Hall, R. W., Lowery, C. L., Nugent, R., \& Mays, G. P. (2010). Late preterm infants: birth outcomes and health care utilization in the first year. Pediatrics, 126(2), e311-e319. https://doi.org/10.1542/peds.2009-2869

Blencowe, H., Cousens, S., Oestergaard, M. Z., Chou, D., Moller, A.-B., Narwal, R., Lawn, J. E. (2012). National, regional, and worldwide estimates of preterm birth rates in the year 2010 with time trends since 1990 for selected countries: a systematic analysis and implications. The Lancet, 379(9832), 2162-2172. https://doi.org/10.1016/S0140-6736(12)60820-4

Brown, M. B., \& Forsythe, A. B. (1974). Robust tests for the equality of variances. Journal of the American Statistical Association, 364-367. https://doi.org/10.1080/01621459.1974.10482955

Dudding, C. C., Brown, D. K., Estis, J. M., Szymanski, C., Zraick, R., \& Former, E. (2019). Best practices in health care simulation. CAPCSD: Council of Academic Programs in Communication Sciences

and

Disorders. 
https://wordpressstorageaccount.blob.core.windows.net/wp-media/wpcontent/uploads/sites/1023/2019/06/eBook-Best-Practices-in-CSD-March-2019.pdf

Eun Uhm, K., Yi, S.-H., Jung Chang, H., Jung Cheon, H., \& Kwon, J.-Y. (2013). Videofluoroscopic swallowing study findings in full-term and preterm infants with dysphagia. Annals of Rehabilitation Medicine, 37(2), 175-182. https://doi.org/10.5535/arm.2013.37.2.175

Ferguson, N. F., Estis, J., Evans, K., Dagenais, P. A., \& VanHangehan, J. (2015). A Retrospective examination of prandial aspiration in preterm infants. Perspectives on Swallowing and Swallowing Disorders (Dysphagia), 24(4), 162. https://doi.org/10.1044/sasd24.4.162

Ferguson, N. F., \& Estis, J. M. (2018). Training students to evaluate preterm infant feeding safety using a video-recorded patient simulation approach. American Journal of SpeechLanguage Pathology, 27(2), 566-573. https://doi.org/10.1044/2017_AJSLP-16-0107

Giambra, B. K., \& Meinzen-Derr, J. (2010). Exploration of the relationships among medical health history variables and aspiration. International Journal of Pediatric Otorhinolaryngology, 74(4), 387-392. https://doi.org/10.1016/j.ijporl.2010.01.010

Glass, H. C., Costarino, A. T., Stayer, S. A., Brett, C., Cladis, F., Davis, P. J., \& Author, A. A. (2015). Outcomes for extremely premature infants. Anesthesia \& Analgesia, 120(6), 13371351. https://doi.org/10.1213/ANE.0000000000000705

Groher, M. E., \& Crary, M. A. (2010). Dysphagia: clinical management in adults and children. Mosby Publishing.

Hamilton, B. E., Martin, J. A., Osterman, M. J. K., Curtin, S. C., \& Matthews, T. J. (2015). Births: Final Data for 2014. National Vital Statistics Reports: Centers for Disease Control and Prevention, National Center for Health Statistics, National Vital Statistics System, 64(12), 1-64. http://www.ncbi.nlm.nih.gov/pubmed/26727629

Holm, S. (1979). Board of the Foundation of the Scandinavian Journal of Statistics A Simple Sequentially Rejective Multiple Test Procedure. Scandinavian Journal of Statistics (6). https://www.ime.usp.br/ abe/lista/pdf4R8xPVzCnX.pdf

Jansen, L. J. (2015). The benefits of simulation-based education. Perspectives on Issues in Higher Education, 18(1), 32-42. https://doi.org/10.1044/ihe18.1.32

Kakodkar, K., \& Schroeder, J. W. (2013). Pediatric Dysphagia. Pediatric Clinics of North America, 60(4), 969-977.https://doi.org/10.1016/j.pcl.2013.04.010

Koenig, J. S., Davies, A. M., \& Thach, B. T. (1990). Coordination of breathing, sucking, and swallowing during bottle feedings in human infants. Journal of Applied Physiology, 69(5), 1623-1629. https://doi.org/10.1152/jappl.1990.69.5.1623 
Lau, C., \& Smith, E. (2012). Interventions to improve the oral feeding performance of preterm infants. Acta Paediatrica, 101(7), e269-e274. https://doi.org/10.1111/j.16512227.2012.02662.x

Lau, C., \& Smith, E. O. (2011). A novel approach to assess oral feeding skills of preterm infants. Neonatology, 100(1), 64-70. https://doi.org/10.1159/000321987

Lefton-Greif, M. A. (2008). Pediatric Dysphagia. Physical Medicine and Rehabilitation Clinics of NA, 19, 837-851. https://doi.org/10.1016/j.pmr.2008.05.007

Liaw, J.-J., Yuh, Y.-S., \& Chang, L.-H. (2005). A preliminary study of the associations among preterm infant behaviors. The Journal of Nursing Research, 13(1), 1-10. http://www.ncbi.nlm.nih.gov/pubmed/15977130

Liu, L., Oza, S., Hogan, D., Chu, Y., Perin, J., Zhu, J., Black, R. E. (2016). Global, regional, and national causes of under-5 mortality in 2000-15: an updated systematic analysis with implications for the Sustainable Development Goals. The Lancet, 388(3027-35). https://doi.org/10.1016/S0140-6736(16)31593-8

Mathew, O. (2011). Apnea of prematurity: pathogenesis and management strategies. Journal of Perinatology, 31(5), 302-310. https://doi.org/10.1038/jp.2010.126

Mercado-Deane, M., Burton, E. M., Harlow, S. A., Glover, A. S., Deane, D. A., Guill, M. F., \& Hudson, V. (2001). Swallowing dysfunction in infants less than 1 year of age. Pediatric Radiology, 31(6), 423-428. https://doi.org/10.1007/s002470100456

Miles, A., Friary, P., Jackson, B., Sekula, J., \& Braakhuis, A. (2016). Simulation-based dysphagia training: teaching interprofessional clinical reasoning in a hospital environment. Dysphagia, 31(3), 407-415. https://doi.org/10.1007/s00455-016-9691-0

Park, J., Thoyre, S., Estrem, H., Associate, P., Pados, B. F., Knafl, G. J., \& Brandon, D. (2016). Mothers' psychological distress and feeding of their preterm infants. The American Journal of Maternal/Child Nursing, 41(4), 221-229. https://doi.org/10.1097/NMC.0000000000000248

Petrou, S. (2005). The economic consequences of preterm birth during the first 10 years of life. An International Journal of Obstetrics \& Gynaecology, 112, 10-15. https://doi.org/10.1111/j.1471-0528.2005.00577.x

Schmitt, J., Arnold, K., Druschke, D., Swart, E., Grählert, X., Maywald, U., Rüdiger, M. (2016). Early comprehensive care of preterm infants-effects on quality of life, childhood development, and healthcare utilization: study protocol for a cohort study linking administrative healthcare data with patient reported primary data. BMC Pediatrics, 16, 104. https://doi.org/10.1186/s12887-016-0640-8 
Shapiro, S. S., \& Wilk, M. B. (1965). An analysis of variance test for normality (complete samples). Biometrika, 52(3-4), 591-611. https://doi.org/10.1093/biomet/52.3-4.591

Thach, B. T. (2007). Maturation of cough and other reflexes that protect the fetal and neonatal airway. Pulmonary Pharmacology \& Therapeutics, 20(4), 365-370. https://doi.org/10.1016/j.pupt.2006.11.011

Thoyre, S. M., \& Carlson, J. (2003a). Occurrence of oxygen desaturation events during preterm infant bottle feeding near discharge. Early Human Development, 72(1), 25-36. https://doi.org/10.1016/s0378-3782(03)00008-2

Thoyre, S. M., \& Carlson, J. R. (2003b). Preterm infants' behavioural indicators of oxygen decline during bottle feeding. Journal of Advanced Nursing, 43(6), 631-641. https://doi.org/10.1046/j.1365-2648.2003.02762.x

Thoyre, S., Shaker, C., \& Pridham, K. (2005). The early feeding skills assessment for preterm infants. Neonatal Network, 24(3), 7-16. https://doi.org/10.1891/0730-0832.24.3.7

Volpe, J. J. (2008). Neurology of the newborn. Saunders/Elsevier.

Ward, E. C., Hill, A. E., Nund, R. L., Rumbach, A. F., Walker-Smith, K., Wright, S. E., Dodrill, P. (2015). Developing clinical skills in paediatric dysphagia management using human patient simulation (HPS). International Journal of Speech-Language Pathology, 17(3), 230-240. https://doi.org/10.3109/17549507.2015.1025846 